\title{
sensors
}

ISSN 1424-8220

(C) 2003 by MDPI

http://www.mdpi.net/sensors

\section{Various Structures Based on Nickel Oxide Thick Films as Gamma Radiation Sensors}

\author{
Khalil Arshak*, Olga Korostynska and Farah Fahim \\ Electronic and Computer Engineering Department, University of Limerick, Plassey Technological \\ Park, Limerick, Ireland. Tel: +353612022 67. Fax: +353613381 76. E-mail khalil.arshak@ul.ie \\ * Author to whom correspondence should be addressed.
}

Received:1 April 2003 / Accepted: 29 April 2003 / Published:30 June 2003

\begin{abstract}
Thick films of Nickel oxide (NiO) in the form of pn-junctions and planar structures with interdigitated electrodes were investigated for $\gamma$-radiation dosimetry purposes. Samples were fabricated using the thick film screen-printing technique. All devices were exposed to a disc-type ${ }^{137} \mathrm{Cs}$ source with an activity of $370 \mathrm{kBq}$. They showed an increase in the values of current with the increase in radiation dose up to a certain level. Performance parameters of the devices, such as sensitivity to $\gamma$-radiation exposure and working dose region, were found to be highly dependant on the composition of the materials used.
\end{abstract}

Keywords: Nickel Oxide; Thick Films; Gamma Radiation; PN-junction; Interdigitated Electrodes

\section{Introduction}

Accurate dosimetry traceable to national standards underpins the protection of the general public and workers across a wide range of activities including general engineering, radiation processing, nuclear power generation, research and use of radiation in medicine and defence. To meet this demand, considerable research into new sensors is underway, including efforts to enhance the sensors performance through both the materials properties and manufacturing technologies. The development of sensors needs to take advantages of the new performances obtained by controlling the physical and chemical properties of the materials.

Metal oxides are widely used in many technological applications, such as coating, catalysis, electrochemistry, optical fibers, sensors, etc [1,2]. Metal oxide sensors have been utilised for several 
decades for their low-cost and simplicity. However, issues with sensitivity, selectivity and stability have limited their use, often in favour of more expensive approaches [3, 4]. The recent availability of various metal oxide materials in high-surface-area nanopowder form, as well as implementation of newly developed nanofabrication techniques, offer tremendous opportunities for sensor manufacturers. New preparation technologies and optimised deposition process are essential to achieve better control of the materials characteristics and consequently to improve the sensor performance. Electrochromic materials, for example Nickel oxide, are used in applications such as energy efficient "smart windows", glare-free and variable reflectance mirrors, high-contrast non-emissive information displays, switchable displays, devices for thermal control, semiconductor-based sensors, etc. [5, 6]. In this work a number of $\mathrm{NiO}$ based thick films of various structures were investigated in terms of their use as gamma radiation sensors. Screen printing technology allows a reliable manufacturing of the sensing elements, with cost-effective mass production and good reproducibility. The detection of radiation was based on the fact, that the properties of the materials undergo changes upon the influence of gamma radiation. Semiconductor nuclear detectors generally employ one of a number of device configurations (planar, sandwich, grid, etc.) [7].

High-energy radiations, such as $\gamma$-rays, change the physical properties of the materials they penetrate. Reactivity of oxide materials is closely connected to the presence of oxygen vacancies [8]. Oxygen vacancies are naturally present in every oxide in the form of Shottky or Frenkel defects, and their concentration can be increased or reduced in several ways [2]. Oxygen vacancies are known as colour centres or F centres (from Farbe, the German word for colour); if the vacancy is localized at the surface a subscript $\mathrm{s}$ is added, $\mathrm{F}_{\mathrm{s}}$. $\mathrm{F}$ centres exist in three states, depending on their electronic charge. Diamagnetic oxygen vacancies can be either neutral, $\mathrm{F}$, or doubly charged, $\mathrm{F}^{2+}$. In the former case two electrons are associated to the vacancy. Of particular interest for the characterization of oxygen vacancies are the paramagnetic $\mathrm{F}^{+}$centres, consisting of a single electron trapped in the cavity formed by removing an oxygen anion, $\mathrm{O}^{-}[2]$. It is believed that ionising radiation causes structural defects (called colour centres) leading to their density change on increase in exposure to $\gamma$-rays [9].

The requirements of radiation sensors include high sensitivity and linear performance over the intended energy range, real-time response, low noise, and acceptable reliability under the exposure conditions [10]. Due to the large number of different applications, there are many alternatives of the radiation sensors. Different materials, geometric arrangements and different physical-detection techniques are used to optimise the trade-offs that exist among the requirements [10].

A number of efforts were devoted to investigate the influence of radiation on the properties of metal oxide materials [11-14]. Gamma irradiation dose of $10^{6}-5 \times 10^{7} \mathrm{rad}$ degraded the electrical and dielectric properties of thin tantalum pentoxide layers obtained by RF sputtering and thermal oxidation in terms of dielectric constant, oxide charge and leakage current [12]. After the electron and $\gamma$-ray radiation of Titanium oxide films prepared by the DC reactive sputtering method, the number of $\mathrm{Ti}^{4+}$ ions decrease in the transition layer, $\mathrm{Ti}^{4+}$ turns to $\mathrm{Ti}^{3+}$ according to the reaction $2 \mathrm{TiO}_{2} \rightarrow \mathrm{Ti}_{2} \mathrm{O}_{3}+\mathrm{O}$ [14]. The influence of ionising radiation on Nickel oxide and its mixture with other oxides prepared by various techniques has been explored [11, 13]. Screen-printed NiO thick films showed decrease in the optical band gap after gamma radiation treatment [11]. Irradiation with 1MGy gamma rays of NiO led to changes in its both surface oxidative abilities and catalytic activities [13]. Deep understanding of 
physical properties of these materials under the influence of radiation exposure is vital for the effective design of dosimeter devices.

In this work values of radiation damage in pn-junctions and interdigitated structures were estimated from changes in the current-voltage characteristics of the manufactured devices. The threshold radiation damage dose depends on the thickness and the structure of the device, properties of the material and on the fabrication technique used.

\section{Experimental Procedure}

Polymer pastes were made of 95 wt.\% of functional materials (pure or Carbon-filled NiO) and 5 wt.\% of $\mathrm{C}_{8} \mathrm{H}_{18} \mathrm{O}_{3}$ with Ethylenglycolmonobutylether as a solvent. Screen printing method (DEK RS 1202 automatic screen printer) was used for the fabrication of various thick film structures. Throughout the experimental work, two different types of devices, namely 1) pn-junctions and 2) interdigitated planar structures were fabricated.

1) $\mathrm{NiO}$ paste was screen-printed on single side polished $\mathrm{N}\langle 111\rangle$ silicon wafers to form pn-junctions having the thickness of $\mathrm{p}$-type layers of $60 \mu \mathrm{m}$.

2) For the fabrication of interdigitated structures microscopic glass slides were used as substrates. Electrical contacts in the form of interdigitated pattern were $15 \mu \mathrm{m}$ in thickness with 10 interdigits on each side and occupied the total area of $8 \times 9 \mathrm{~mm}^{2}$. Commercial DuPont 4929 silver paste was used to manufacture the electrical contacts for all the devices. On top of them, radiation-sensitive layers of $\mathrm{NiO}-$ based polymer pastes (pure $\mathrm{NiO}, 0.1 \mathrm{wt} \%$ and $0.2 \mathrm{wt} \%$ Carbon-filled $\mathrm{NiO}$ ) having thickness of $60 \mu \mathrm{m}$ were printed. Adding various amount of Carbon powder into the pastes controls the conductivity of the films.

A disc type ${ }^{137} \mathrm{Cs}$ radiation source with activity of $370 \mathrm{kBq}$ was used for exposing the samples to $\gamma$ radiation at room temperature. A set of irradiations were performed changing the exposure time and hence the dose. During the irradiation to $\gamma$-rays, external environmental conditions, such as temperature, humidity and light illumination, were maintained constant to ensure they do not contribute to the response characteristic of these devices.

\section{Results and Discussion}

\section{Structural Properties of NiO Thick Films}

Figure 1 shows the XRD patterns of screen-printed NiO thick film along with the list of standard $\mathrm{XRD}$ peaks for $\mathrm{NiO}$ [15]. The XRD data highlighted that $\mathrm{NiO}$ thick films possess crystalline structure and exhibited the characteristic peaks of rock salt NiO. A small deviation of the peaks from the standard [15] is attributed to the material preparation technique used.

Since XRD technique provides an average structure for the crystalline phases with long-range structural order, the Scanning Electron Microscope (SEM) analysis of the samples was performed using Jeol JSM-840 SEM to reveal the morphology of the NiO films. Figure 2 illustrates SEM micrographs of $\mathrm{NiO}$ thick films with magnifications of 1000 and 4000 respectively. It demonstrates the 
porous surface morphology of these films that consist of the grains with variable sizes in the range of $1-10 \mu \mathrm{m}$.

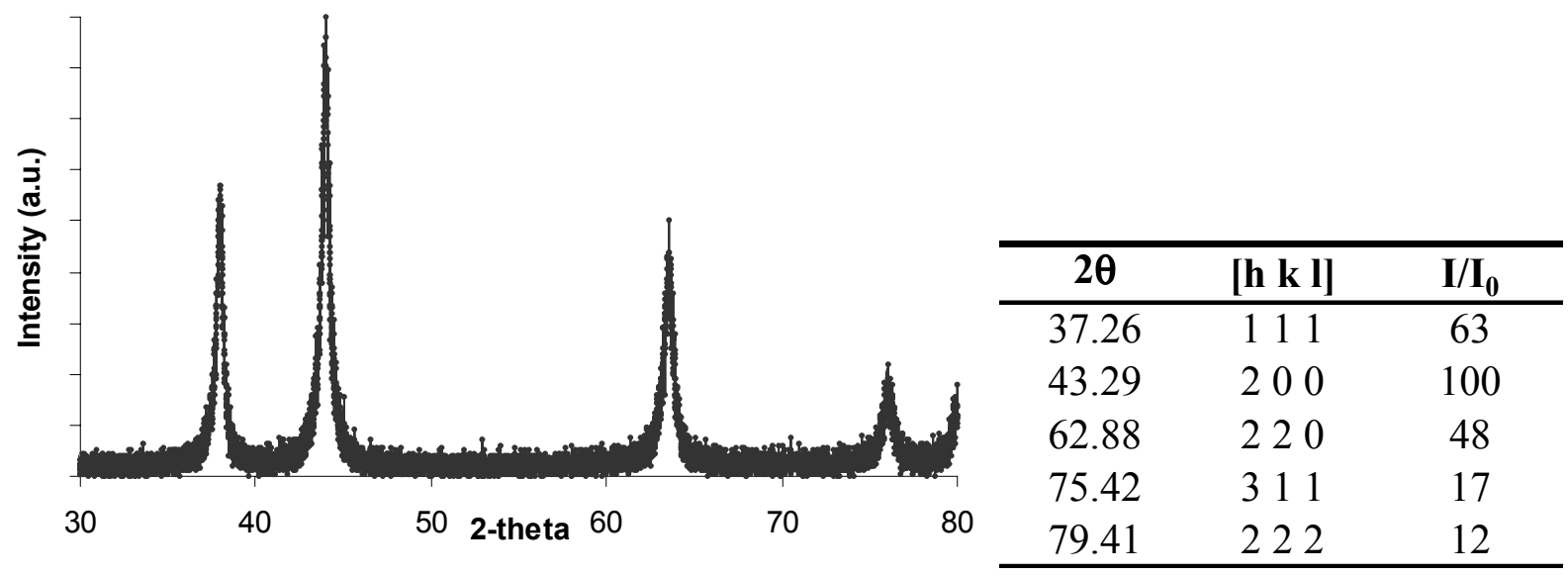

Figure 1. XRD patterns of screen-printed $\mathrm{NiO}$ thick film and standard XRD peaks for NiO.
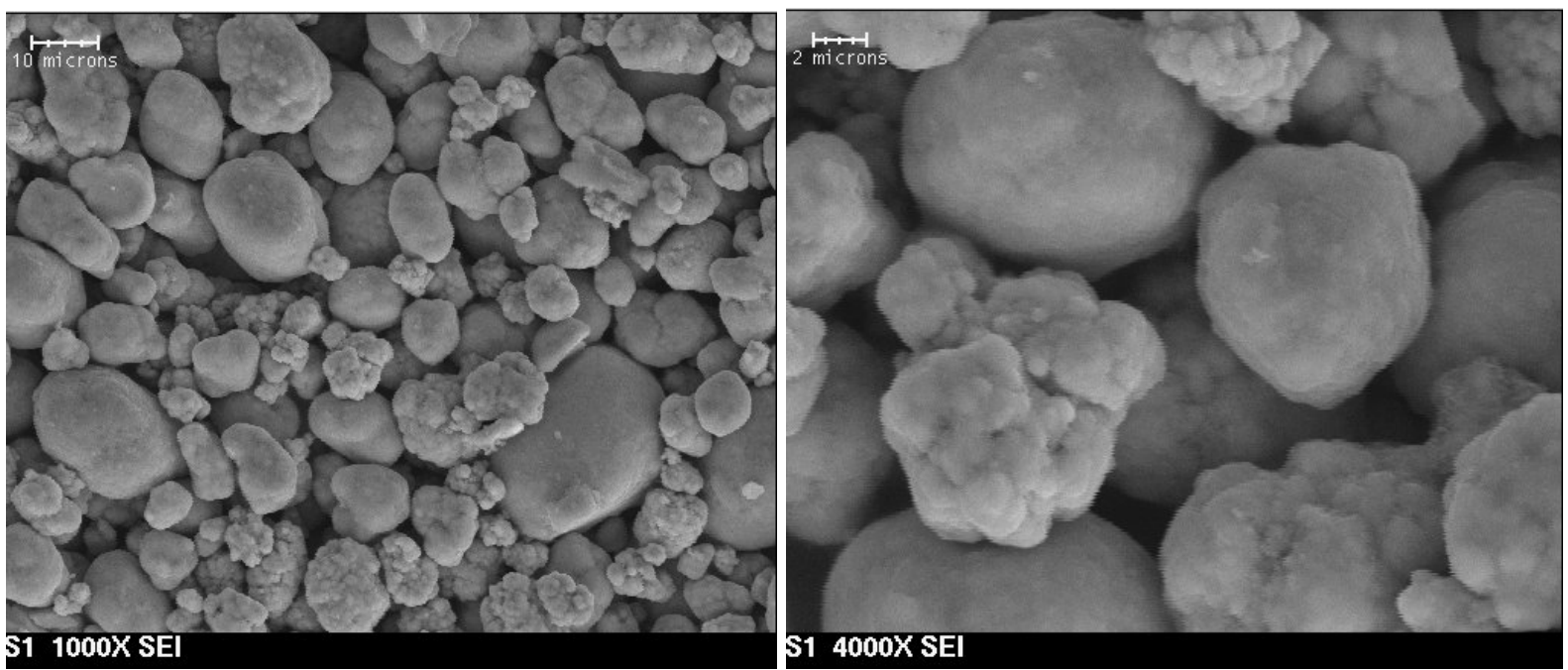

Figure 2. SEM images of $\mathrm{NiO}$ thick film with magnifications of 1000 and 4000 respectively.

\section{Effect of $\gamma$-radiation on NiO Diode Structures}

Figure 3 shows typical current-voltage characteristics of $\mathrm{NiO} / \mathrm{Si}$ samples, as-printed and $\gamma$-irradiated with different doses. Figure 4 demonstrates more clearly I-V plots for these devices under the reversebiased conditions. In all graphs below, the exposed radiation doses are given in $\mu \mathrm{Sv}$ units.

The values of both, the leakage current under the reverse-biased condition and the values of current when the diode is forward-biased, are increased considerably with the increase in radiation dose up to a level of $511 \mu \mathrm{Sv}$. The parameter controlling leakage is the generation lifetime, which governs the rate of emission of holes in the depletion region [16]. To trace the radiation-induced changes in the values of current under the applied voltages of $+6 \mathrm{~V}$ and $-9 \mathrm{~V}$ see Figure 5. It illustrates the dependences of normalized currents $\left(\mathrm{I}-\mathrm{I}_{0}\right) / \mathrm{I}_{0}$ with dose for $\mathrm{NiO} / \mathrm{Si}$ diodes. These curves may roughly be considered to 
consist of 3 regions. Linear increase in the values of currents was monitored with the increase in radiation to a dose of around $220 \mu \mathrm{Sv}$. In the second region from $220 \mu \mathrm{Sv}$ to around $340 \mu \mathrm{Sv}$ a faster increase in the values of currents was observed. Further increase in radiation dose led to some kind of saturation in I-V plots.

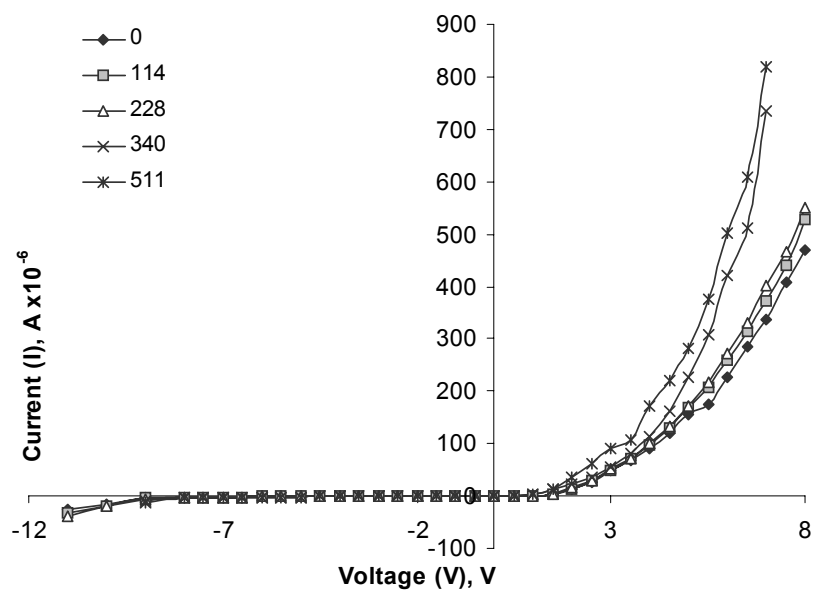

Figure 3. Full-scale I-V plots of as-printed and $\gamma$-irradiated at different doses $\mathrm{NiO} / \mathrm{Si}$ pnjunctions.

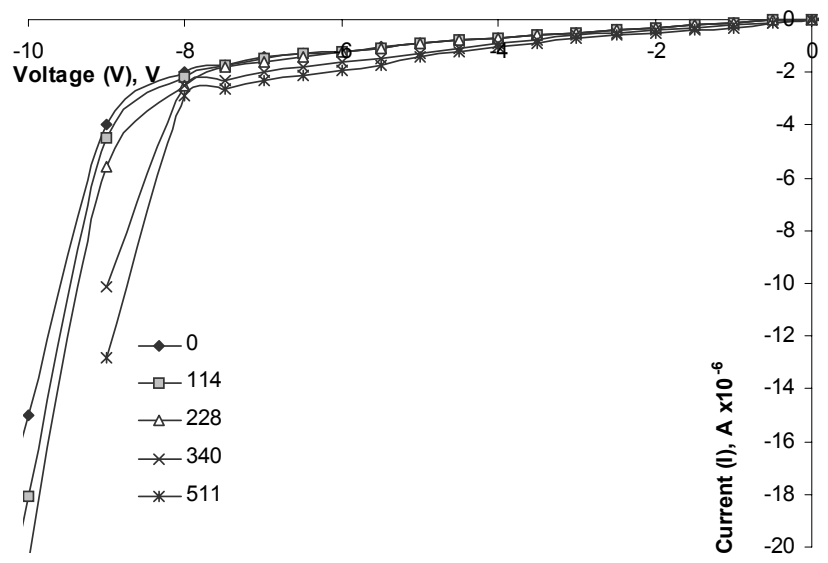

Figure 4. $\mathrm{I}-\mathrm{V}$ plots of as-printed and $\gamma$-irradiated $\mathrm{NiO} / \mathrm{Si}$ diodes under the reverse-bias.

The current increase was caused by the grain boundaries, which serve as current paths resulting in poor leakage current characteristics. Another reason for the worsening of the electrical properties is that the films were damaged by the creation of radiation defects in the form of broken Ni-O bonds. It is reasonable to assume that the increase in the leakage current after the influence of $\gamma$-radiation is partially attributed to the lowering of the barriers height at $\mathrm{Ag} / \mathrm{NiO}$ interface. Similar effect of $\gamma$-rays was observed in $\mathrm{Al} / \mathrm{Ta}_{2} \mathrm{O}_{5}$ interface because of building up a charge in $\mathrm{Ta}_{2} \mathrm{O}_{5}$ near this contact [12]. During irradiation process modification of $\mathrm{Si}-\mathrm{NiO}$ interface takes place as a result of oxidation of $\mathrm{Si}$ wafer, leading to the enlargement of the mixed transition region, where $\mathrm{SiO}_{2}$ and the intermediate oxidation states of $\mathrm{Si}$ coexist. The latent defects, which are activated during irradiation, are in the form of oxide traps and are also responsible for deterioration of the device characteristics [12]. 


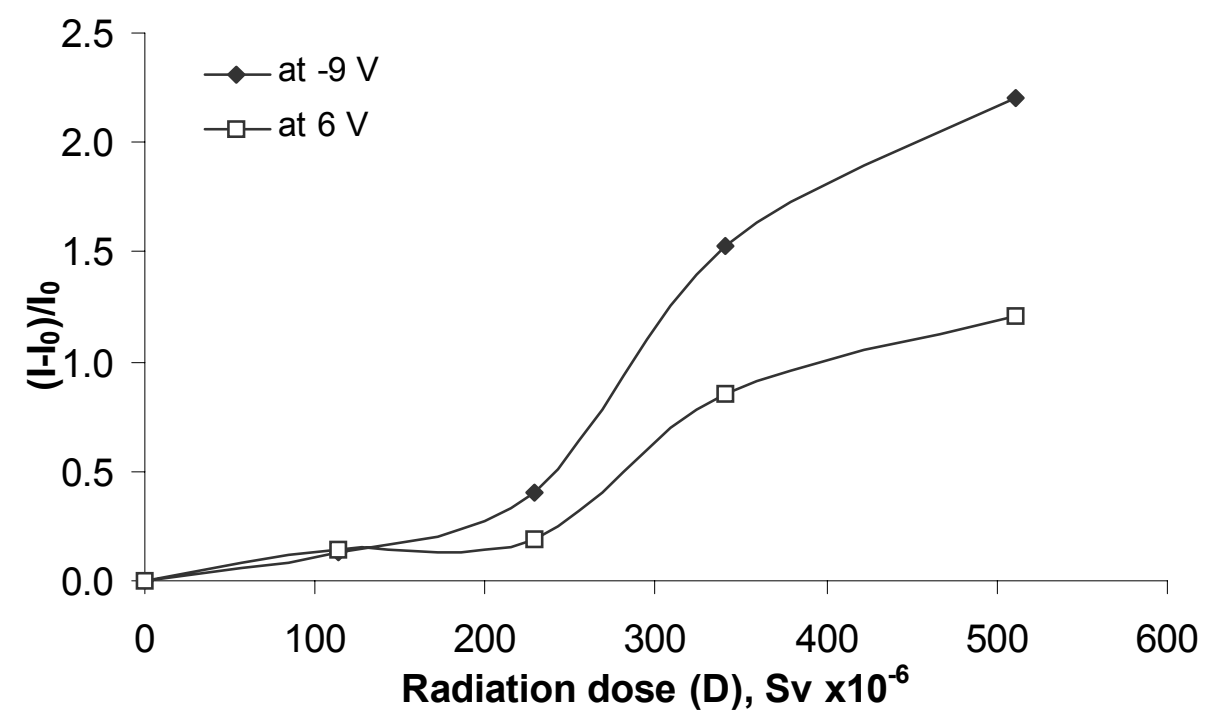

Figure 5. Dependence of normalized current $\left(\mathrm{I}-\mathrm{I}_{0}\right) / \mathrm{I}_{0}$ with dose under the applied voltages of $-9 \mathrm{~V}$ and $+6 \mathrm{~V}$ for $\mathrm{NiO} / \mathrm{Si}$ pn-junction structures.

\section{Effect of $\gamma$-radiation on Carbon-Filled NiO Planar Structures with Interdigitated Electrodes}

In this work electrodes with interdigitated patterns were used to maximise the electrical admittance between two conducting structures in order to exploit the radiation-induced changes in the electrical properties of the materials printed over them.

Figure 6 shows plots of the I-V characteristics that were recorded for as-printed and $\gamma$-irradiated $\mathrm{NiO}$ planar structures with interdigitated electrodes. One can see that irradiation of the samples resulted in the significant alteration of current-voltage characteristics. Figure 7 depicts these changes, showing the increase in the values of normalized current $\left(\mathrm{I}-\mathrm{I}_{0}\right) / \mathrm{I}_{\mathrm{o}}$ with the increase in radiation dose up to $171 \mu \mathrm{Sv}$ under the applied voltages of $3 \mathrm{~V}$ and $10 \mathrm{~V}$.

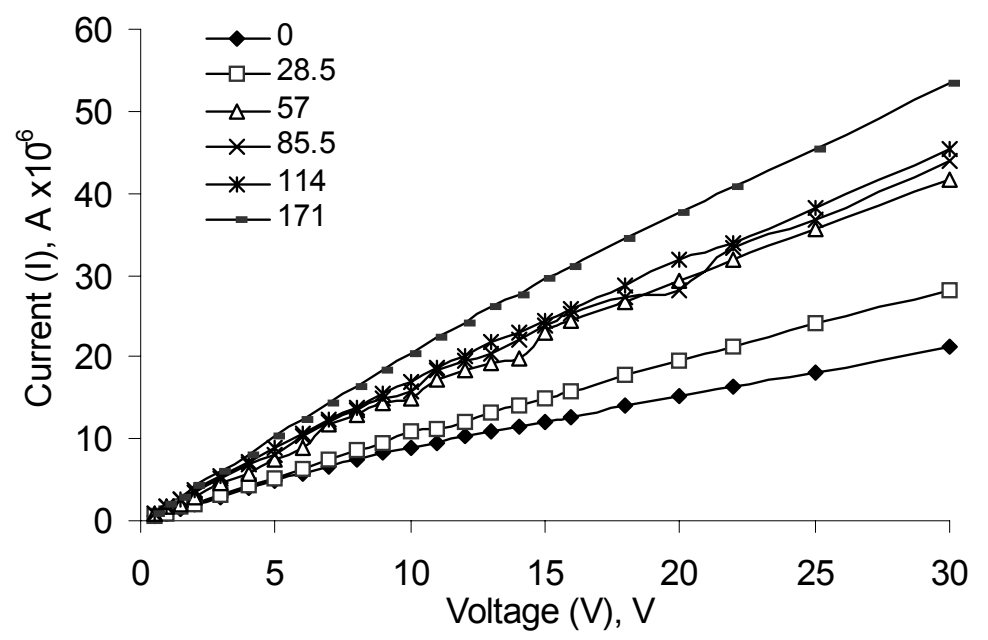

Figure 6. I-V plots of as-printed and $\gamma$-irradiated at different doses $\mathrm{NiO}$ planar structures with interdigitated electrodes. 


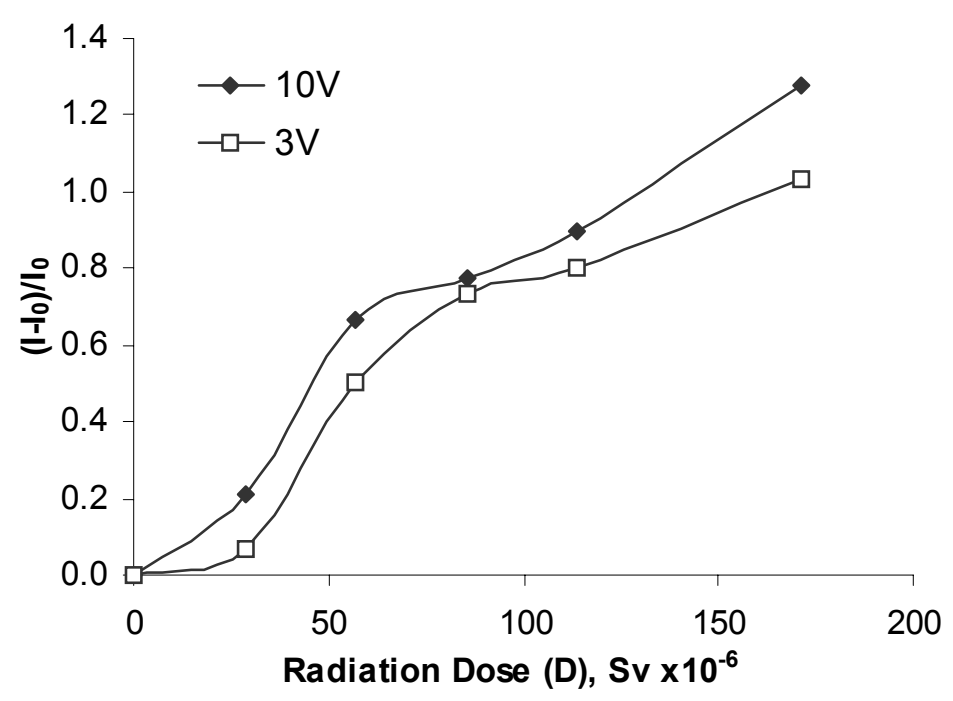

Figure 7. Plot of normalized current $\left(\mathrm{I}-\mathrm{I}_{0}\right) / \mathrm{I}_{0}$ with dose under the applied voltages of $10 \mathrm{~V}$ and $3 \mathrm{~V}$ for $\mathrm{NiO}$ interdigitated planar structures.

To explore the effect of the composition on the performance of these devices, Carbon doping of $0.1 \mathrm{wt} . \%$ and $0.2 \mathrm{wt} . \%$ was used to increase the conductivity of polymer pastes. Increased conductivity of the films results in more pronounced signal response to external influences, such as $\gamma$-rays. As well it is beneficial from the circuitry point of view as it increase the signal/noise ratio and provides more reliable data output.

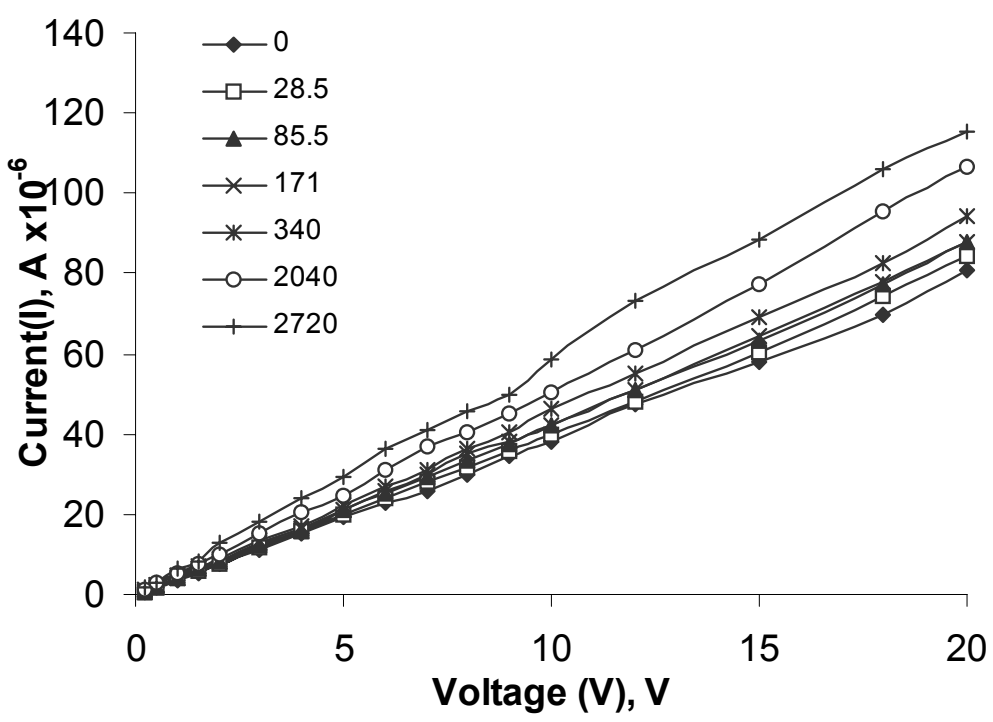

Figure 8. I-V plots of as-printed and $\gamma$-irradiated $\mathrm{NiO}$ planar structures doped with $0.1 \mathrm{wt} . \%$ of C.

Figure 8 shows typical plots of the I-V characteristics of as-printed and $\gamma$-irradiated $\mathrm{NiO}$ planar structures doped with $0.1 \mathrm{wt} . \%$ of Carbon. Figure 9 shows the dependence of the normalized current $\left(\mathrm{I}-\mathrm{I}_{0}\right) / \mathrm{I}_{\mathrm{o}}$ with the increase in radiation dose up to $2720 \mu \mathrm{Sv}$ under the applied voltages of $1 \mathrm{~V}$ and $8 \mathrm{~V}$. 


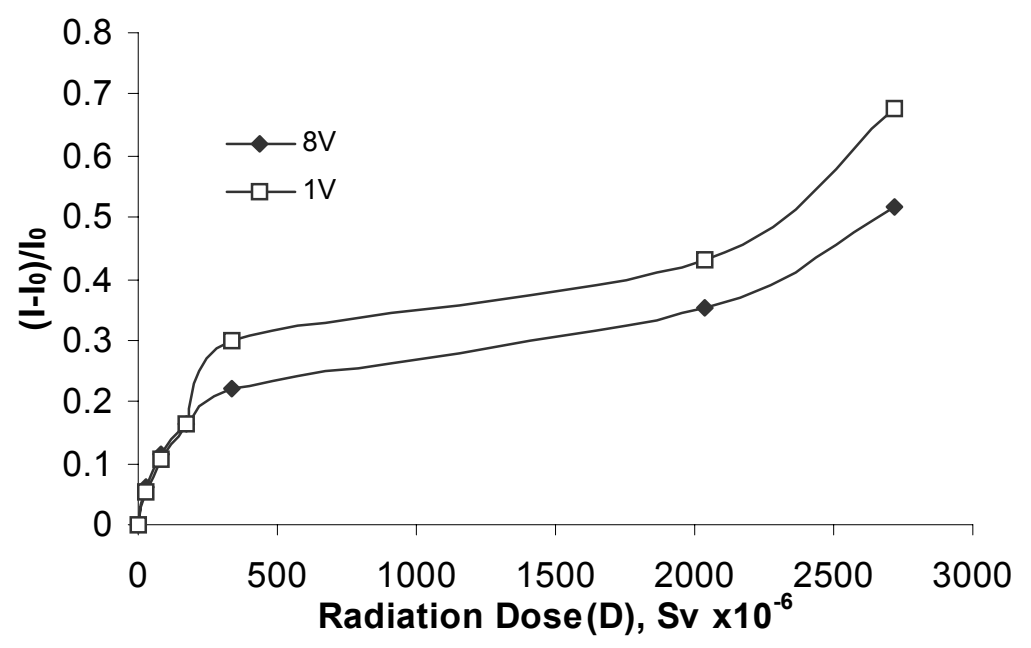

Figure 9. Plots of normalised current $\left(\mathrm{I}-\mathrm{I}_{0}\right) / \mathrm{I}_{0}$ versus dose under the applied voltages of $1 \mathrm{~V}$ and $8 \mathrm{~V}$ for $0.1 \mathrm{wt} . \%$ Carbon-filled $\mathrm{NiO}$ planar structures.

Carbon doping of $\mathrm{NiO}$ thick films of $0.1 \mathrm{wt} . \%$ resulted in larger increments in the values of current with the increase in radiation dose compared to the counterpart of pure $\mathrm{NiO}$ thick film structures. Moreover, this increase in values of current continued to be substantial at higher radiation doses thereby increasing its working range to $2720 \mu \mathrm{Sv}$ compared to $171 \mu \mathrm{Sv}$ of pure $\mathrm{NiO}$ samples.

Further increase in the level of Carbon doping makes the samples too conductive. Figure 10 shows plots of the I-V characteristics of as-printed and $\gamma$-irradiated $\mathrm{NiO}$ planar structures doped with $0.2 \mathrm{wt} . \%$ of Carbon. Figure 11 shows the dependence of the normalized current $\left(I-I_{0}\right) / I_{0}$ with the increase in radiation dose up to $1700 \mu \mathrm{Sv}$ under the applied voltages of $0.1 \mathrm{~V}$ and $0.3 \mathrm{~V}$ for $0.2 \mathrm{wt} . \%$ Carbonfilled $\mathrm{NiO}$ planar structures with interdigitated electrodes. While the applied voltages were relatively low, the values of current were high, for example at the applied voltage of $0.8 \mathrm{~V}$ the value of current reached $3.5 \mathrm{~mA}$. However such a device can find an application in low power supply conditions, for example in personal dosimetry. The substantial signal response has a trade-off in lowering the working radiation range of the devices.

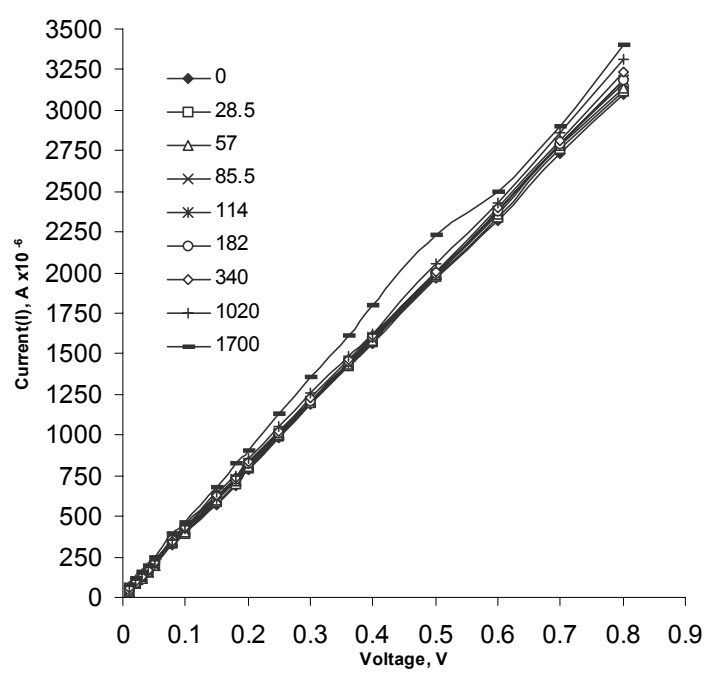

Figure 10. I-V plots of as-printed and $\gamma$-irradiated at different doses $\mathrm{NiO}$ interdigitated planar structures doped with 0.2 wt. $\%$ of $C$. 


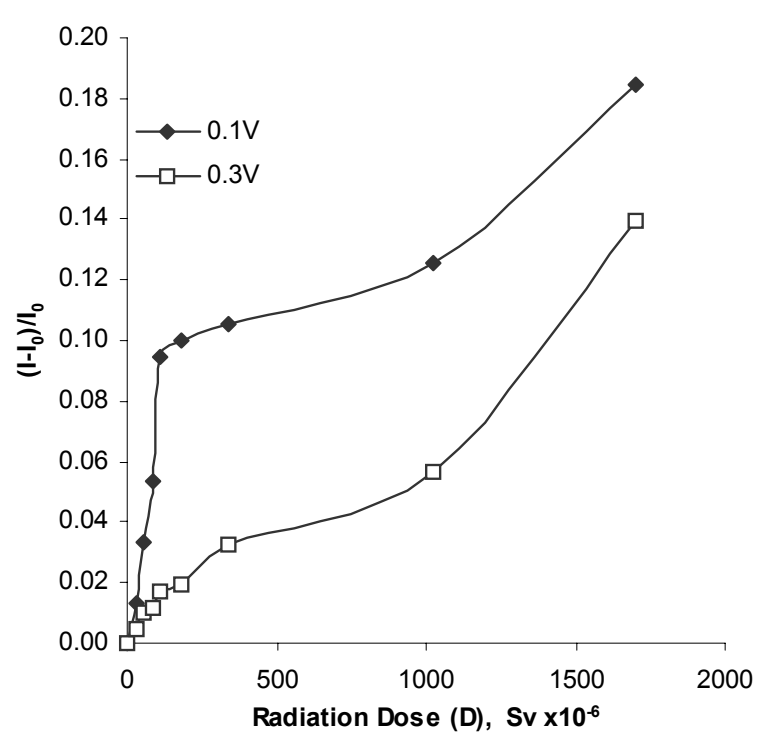

Figure 11. Plots of normalized current $\left(\mathrm{I}-\mathrm{I}_{0}\right) / \mathrm{I}_{0}$ with dose under the applied voltages of $0.1 \mathrm{~V}$ and $0.3 \mathrm{~V}$ for 0.2 wt.\% Carbon-filled $\mathrm{NiO}$ planar structures.

The $\gamma$-radiation-induced changes in the electrical properties of all the samples were found to be similar to the dose response of most materials used in thermoluminescence (TL) dosimetry [17]. They usually show a linear, then supralinear, followed by saturating response and further increase in dose leads to their damage.

The details of the experimentally measured dose response depend on a great number of instrumental, radiation field and material parameters [17]. Similar feature of dose response was found in TL materials and lies in the dependence of the supralinearity on ionisation density, i.e., on gamma ray energy and on the type of radiation. The value of maximum supralinearity rapidly decreased with decreasing gamma ray energy: for $1.25 \mathrm{MeV}$ gamma rays in $\mathrm{LiF}: \mathrm{Mg}, \mathrm{Cu}, \mathrm{P}$ showed no supralinearity [17]. The difference in the properties of the materials used emerges as reasonable explanation to the changes of supralinear region in dependence of electrical parameters on radiation dose. However, the region of linear response is preferable in radiation dosimetry.

The model for colour centre kinetics assumes that the level of the radiation damage should be dose rate dependent because of the damage recovery [18]. As colour centres are created under irradiation, they also annihilate even under the room temperature. During irradiation, both annihilation and creation coexist; the colour centre density will reach equilibrium at a level depending on the dose rate applied. The creation and annihilation constants can be determined by using experimental data obtained under one particular dose rate, and can then be used to predict the behaviour of the same sample under different dose rates [18]. The threshold radiation damage dose depends on the thickness and the structure of the device, properties of the material and the fabrication technique used.

\section{Conclusion}

The possibility of using pure and Carbon-filled $\mathrm{NiO}$ thick films as sensitive materials to gamma radiation was explored. Various devices in the forms of pn-junctions and interdigitated planar 
structures were manufactured using screen-printing thick film technique. All devices were exposed to a disc-type ${ }^{137} \mathrm{Cs}$ source with an activity of $370 \mathrm{kBq}$. They showed an increase in the values of current with the increase in radiation dose up to a certain level, which could be considered as a working region. For $\mathrm{NiO} / \mathrm{Si}$ pn-junction, the values of both the leakage current under the reverse-biased conditions and the values of current when the diode was forward-biased increased considerably with the increase in radiation dose up to a level of $511 \mu \mathrm{Sv}$.

The electrical properties of the films were highly dependant on the composition of the oxide materials used. It was experimentally demonstrated that it is possible to fabricate a device that would satisfy the requirement of particular application, in this case the sensitivity to $\gamma$-radiation exposure and working dose region. Planar $\mathrm{NiO}$ thick film structures with interdigitated electrodes showed increase in the values of current with the increase in radiation dose up to $171 \mu \mathrm{Sv}$. Carbon doping of NiO thick films of $0.1 \mathrm{wt} . \%$ resulted in larger increments in the values of current and increasing the working range to $2720 \mu \mathrm{Sv}$. NiO planar structures doped with $0.2 \mathrm{wt} . \%$ of Carbon showed the increase in the values of current with the increase in radiation dose up to $1700 \mu \mathrm{Sv}$. The substantial signal response has a trade-off in lowering the working radiation range of the devices.

All these devices might be regarded as a cost-effective alternative for room temperature real time gamma radiation dosimetry.

\section{Acknowledgments}

This work was performed with the assistance of AMT Ireland at University of Limerick, as part of the RADSENAD project (ref. PRP00/AMT/06), sponsored by Enterprise Ireland Development Funds.

\section{References}

1. Heinrich, V. E.; Cox, P. A. The surface science of metal oxides; Cambridge University Press: Cambridge, 1994.

2. Pacchioni, G. Ab initio theory of point defects in oxide materials: structure, properties, chemical reactivity. Solid State Sciences 2000, 2, 161-179.

3. Faia, P. M.; Pereira, M. A.; Nunes, A. M.; Furtado, C. S. Electronic noses, a different approach to the sensitivity and selectivity issues. Journal of the European Ceramic Society 1999, 19, 883-886.

4. Hockensmith, C. M.; Goldsby, J. C.; Kacik, T. Thermal studies of new precursors to indium-tin oxides for use as sensor materials in the detection of $\mathrm{NO}_{\mathrm{x}}$. Thermochimica Acta 1999, 340-341, 315-322.

5. Ragan, D. D.; Svedlindh, P.; Granqvist, C. G. Electrochromic Ni oxide films studied by magnetic measurements. Solar Energy Materials and Solar Cells 1998, 54, 247-254.

6. Neubecker, A.; Pompl, T.; Doll, T.; Hansch, W.; Eisele, I. Ozone-enhanced molecular beam deposition of nickel oxide (NiO) for sensor applications. Thin Solid Films 1997, 310, 19-23.

7. Schlesinger, T. E.; Toney, J. E.; Yoon, H.; Lee, E. Y.; Brunett, B. A.; Franks, L.; James, R. B. Cadmium zinc telluride and its use as a nuclear radiation detector material. Materials Science and Engineering: R: Reports 2001, 32, 103-189. 
8. Pacchioni, G.;Pescarmona, P. Structure and stability of oxygen vacancies on sub-surface, terraces, and low-coordinated surface sites of MgO:: an ab initio study. Surface Science 1998, 412-413, 657671.

9. Zhu, R. y. Radiation damage in scintillating crystals. Nuclear Instruments and Methods in Physics Research Section A: Accelerators, Spectrometers, Detectors and Associated Equipment 1998, 413, 297-311.

10. Audet, S.; Steigerwald, J. In Semiconductor Sensors; edited by Sze, S. M., Ed.; John Wiley \& Sons, Inc.: New York, Chichester, Brisbane, Toronto, Singapore, 1994; Vol. pp. 271-329.

11. Arshak, K., Korostynska, O., and Harris, J. Gamma radiation dosimetry using screen printed nickel oxide thick films. Proceeding of MIEL 2002, 23rd International conference on Microelectronics, 357-360 (2002).

12. Atanassova, E.; Paskaleva, A.; Konakova, R.; Spassov, D.; Mitin, V. F. Influence of $\gamma$ - radiation on thin $\mathrm{Ta}_{2} \mathrm{O}_{5}$-Si structures. Microelectronics Journal 2001, 32, 553-562.

13. Mucka, V.; Podlaha, J.; Silber, R. NiO-ThO 2 mixed catalysts in hydrogen peroxide decomposition and influence of ionizing radiation. Radiation Physics and Chemistry 2000, 59, 467-475.

14. Zhang, J. D.; Fung, S.; Li-Bin, L.; Zhi-Jun, L. Ti ion valence variation induced by ionizing radiation at $\mathrm{TiO}_{2} / \mathrm{Si}$ interface. Surface and Coatings Technology 2002, 158-159, 238-241.

15. Powder Diffraction File, Joint Committee on Powder diffraction, International Center for Diffraction Data, Swarthmore, PA, 1987, Card. 4-0835.

16. Holmes-Siedle, A. G.; Adams, L. Handbook of radiation effects; Oxford University Press: Oxford; New York, 1993.

17. Horowitz, Y. S. Theory of thermoluminescence gamma dose response: The unified interaction model. Nuclear Instruments and Methods in Physics Research Section B: Beam Interactions with Materials and Atoms 2001, 184, 68-84.

18. Deng, Q.; Yin, Z.; Zhu, R. Y. Radiation-induced color centers in La-doped $\mathrm{PbWO}_{4}$ crystals. Nuclear Instruments and Methods in Physics Research Section A: Accelerators, Spectrometers, Detectors and Associated Equipment 1999, 438, 415-420.

Sample Availability: Available from the authors.

(C) 2003 by MDPI (http://www.mdpi.net). Reproduction is permitted for noncommercial purposes. 